\title{
Temporal trimming: Evidence that common-onset masking shortens perceptual sampling of conscious object representations
}

\author{
Geoffrey W. Harrison ${ }^{1}$ - Jason Rajsic ${ }^{2}$ • Daryl E. Wilson ${ }^{1}$
}

Published online: 17 July 2017

(C) The Psychonomic Society, Inc. 2017

\begin{abstract}
Common-onset masking (COM) refers to a methodology where a mask can impair awareness of an object if the mask's offset is delayed relative to the offset of the object. This method has classically been used to understand how discontinuities in visual input lead to the discrete removal of object representations before they reach conscious awareness. However, COM has recently been shown to reduce the precision of conscious object representations (Harrison, Rajsic, \& Wilson, Psychonomic Bulletin \& Review, 23(1), 180-186, 2016). As a result, Harrison et al. proposed that COM shortens the temporal window for perceptual sampling of an object's representation, an account consistent with interruption-based theories of masking. In the present study we modified the standard COM methodology to assess the impact of a delayed mask offset on the temporal perception of an object's representation. Across two experiments we provide novel evidence that a delayed mask offset can impair temporal perception of a conscious percept, such that it reduces the percept's perceived duration (Experiment 1), and prematurely terminates updating of the percept's dynamic orientation (Experiment 2). We refer to these results as temporal trimming, and suggest that the mechanism responsible for COM operates during the sustained perception of an object.
\end{abstract}

Keywords Temporal processing · Visual awareness · Visual perception

\section{Geoffrey W. Harrison}

8gh3@queensu.ca

1 Psychology, Queen's University, Kingston, Canada

2 Psychology, University of Toronto, Toronto, Canada

\section{Introduction}

One highly influential tool used to investigate how the visual system resolves discontinuity in its input during the initial stages of object-based perception has been common-onset masking (COM; Di Lollo et al., 2000; Goodhew, Pratt, Dux, \& Ferber, 2013; Moore \& Lleras, 2005). In COM, four small dots (the mask) are presented surrounding a target object. Even when the target is presented for a brief duration $(<50$ $\mathrm{ms}$ ) if the target and mask offset together, awareness of the target is easily obtained. In contrast, if the offset of the mask is delayed relative to the offset of the target, target awareness is greatly reduced (Di Lollo et al., 2000). Leading theories of COM explain these failures in target awareness via a mechanism that resolves visual discontinuity during the formation of an object's representation. The initial presentation of the target and mask at the same time and in the same spatial location begins their encoding as a unified object. When both target and mask offset together, the lack of any discontinuity allows their consolidated representation to reach awareness unabated. Conversely, in delayed mask-offset conditions, target information is presented only briefly relative to the mask. In these cases, the initial presence of target information is treated as a discontinuous event that is resolved by its removal before the target information reaches awareness. Classically, the removal of target information in COM has been treated as an all-ornone process (Di Lollo et al., 2000; Lleras \& Moore, 2003). However, recent research by Harrison et al. (2016; see also: Salahub \& Emrich, 2016), has challenged the discrete removal of target information, calling for a revision of theories of COM and, more generally, how the brain resolves discontinuity in visual input during object perception.

Harrison and colleagues (2016) showed that COM can reduce the precision of a target's representation, thus providing evidence that COM does not necessarily impact target 
representation in a discrete, all-or-none fashion. They adapted a continuous response task and a mixture modeling analysis approach (Bays \& Husain, 2008; Zhang \& Luck, 2008) to a standard COM paradigm and showed that four-dot masking can both increase random guessing and reduce the representational precision of a target's orientation. To account for the precision decrement caused by the four-dot mask, Harrison et al. (2016) proposed that the resolution of discontinuity during delayed mask offset trials in COM does not simply remove target information from the initial object representation, but instead shortens the temporal window for perceptual sampling of target information, leading to noisier representations of target features. On trials in which the sampling window is greatly reduced, not enough information is acquired for the target's representation to reach awareness; consequently, these trials lead to an increase in random guessing about a target's orientation. On other trials in which the sampling window is only slightly reduced, the target's representation will reach awareness, but with a weaker representation compared to simultaneous mask-offset trials. These latter trials underlie the finding that $\mathrm{COM}$ can reduce the precision of target representation. Explaining masking via an interruption of a spatial sampling process is not new (Spencer \& Shuntich, 1970), but was previously dismissed as an explanation of COM due to the distinct profile with which the mask impacts target awareness over time (Enns, 2004; but see Breitmeyer \& Öğmen, 2006). Critically, if the revised theory of a shortened perceptual sampling window during COM is correct, it generates an important new prediction about the visual system's method for resolving conflict in object-based processing: events that signal discontinuity about an object should impact the perceived duration of that object's features even after those features have clearly reached awareness.

To our knowledge, all studies of COM to date have examined the effects of COM on the physical characteristics of a target object (e.g., shape, color). ${ }^{1}$ In order to assess the impact of COM on target features that have reached awareness, we need to increase the presentation duration of targets well beyond the standard range of 10-50 ms. In doing so, we can no longer assess the perception of a target's static physical features, as the assessment of those features is necessarily confounded with those features being already encoded into shortterm memory. Thus, we need a target feature that requires continuous updating over time. For static objects, an obvious feature that meets this constraint is perceived duration. If the mechanism responsible for COM shortens the window over which perceptual information for a target is sampled, then a delayed mask offset should prematurely terminate the visual

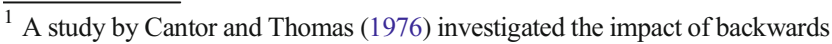
masking on the perceived duration of a target. However, this study used brief target presentations $(<70 \mathrm{~ms})$ and provided conflicting results on the effect of masking on perceived duration across two stimulus types.
}

information for a target that reaches awareness. This premature termination of target information should result in a reduction of the target's perceived duration.

In the current experiment, to test whether a delayed mask offset reduces the perceived duration of a target object, we used a duration judgment task (adapted from the temporal order judgment (TOJ) task (Allik \& Kreegipuu, 1998; Shore, Spence, \& Klein, 2001)) in which one target was presented for a standard duration and a second target was presented for a range of durations - some shorter and some longer than the standard. The psychophysical function relating the duration judgment as a function of the temporal offset of the two targets allows for an estimation of the point of subjective equality (PSE) - the point at which the two targets are perceived to be of equal duration. In the Experimental condition, participants were presented with eight objects around fixation with two target objects surrounded by four dot masks. The participant's task was to judge the relative duration of the two targets. On every trial, one mask was a simultaneous mask (mask's offset simultaneous with its target), while the other mask was a delayed mask (mask's offset delayed relative to its target). If delaying the offset of a four-dot mask reduces the perceived duration of that target, then the PSE for the Experimental condition should be shifted. Specifically, a shift in the PSE in the Experimental condition would reflect the additional duration that a target with a delayed mask needs to be presented in order for it to have been perceived as having an equal duration as a target with a simultaneous mask.

\section{Experiment 1}

\section{Method}

\section{Participants}

Twenty Queen's University students participated for financial compensation. All were volunteers, had normal or correctedto-normal vision, and were naïve to COM and the experiment's purpose.

\section{Apparatus}

The experiment was conducted on a personal computer in a dimly-lit room. Stimuli were presented with Psychophysics Toolbox version 3.0.8 (Brainard, 1997) in Matlab on a 16in. CRT monitor. Participants responded using a keyboard.

\section{Design and procedure}

The experiment consisted of 12 blocks of 144 trials for a total of 1,728 experimental trials, preceded by two blocks of 20 practice trials. An example of the stimulus presentation screen 
is provided in Fig. 1. Each trial began with the presentation of a small white fixation point (radius $0.15^{\circ}$ ) at the center of a dark-gray background $\left(17 \mathrm{~cd} / \mathrm{m}^{2}\right)$ for $500 \mathrm{~ms}$. This was followed by the presentation of eight shapes on an imaginary circle (radius $3.5^{\circ}$ ) such that each shape was equidistant from the fixation point. The targets were always two diamonds (approximately $0.5^{\circ}$ ) amongst six other shapes (two squares, two circles, and two triangles, all approximately $0.5^{\circ}$ ). To facilitate target detection, the two diamonds were drawn in a slightly brighter white $\left(62 \mathrm{~cd} / \mathrm{m}^{2}\right)$ than the non-target shapes, the masks, and the fixation point $\left(51 \mathrm{~cd} / \mathrm{m}^{2}\right)$. One target's location was selected randomly from $10-169^{\circ}$ along the imaginary circle and the second target was always presented at the opposite location $\left(+180^{\circ}\right)$. This separation of the targets ensured that the targets could easily be identified as belonging to the left or right side of the screen. The remaining six shapes were positioned such that the two targets and the six nontargets were equally spaced on the imaginary circle $\left(45^{\circ}\right.$ apart). In the Experimental condition trials, the two diamonds (the targets) were surrounded by four small white dots (each approximately $0.1^{\circ}$ ), which made up the corners of an imaginary square (centered $3.5^{\circ}$ from fixation). In the Control condition trials, the two masks were again $180^{\circ}$ apart but surrounded two of the non-target shapes. The six non-target shapes had offsets $240 \mathrm{~ms}$ after presentation onset. One of the target diamonds, referred to as the standard target, also had an offset $240 \mathrm{~ms}$ after presentation onset. The other target is referred to as the varied target as its offset was varied relative to the standard target by one of nine Temporal Offset values $(-136,-102,-68,-34,0,34,68,102,136 \mathrm{~ms}$, where 0 corresponds to the standard 240-ms offset). One of the masks had a simultaneous offset with its associated target (simultaneous mask) and the other mask's offset was always delayed by $300 \mathrm{~ms}$ relative to its target (delayed mask). Participants made a duration judgment such that half were instructed to respond as to whether the left (using the ' $\mathrm{z}$ ' key) or right (using the ' $\mathrm{m}$ ' key) target was presented for a shorter duration and half responded as to which target was presented for a longer duration (Fig. 2).

All combinations of Temporal Offset values, whether they were applied to the left or right target and whether they were applied to the target that was paired with a simultaneous or

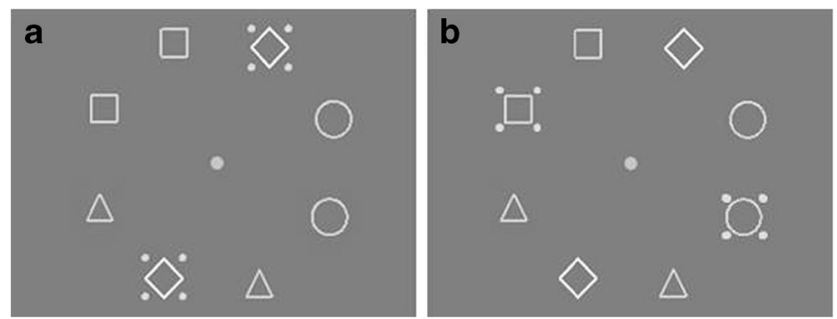

Fig. 1 Example initial stimulus presentations for Experiment 1 (Panel A: Experimental condition; Panel B: Control condition). See Method section for details on trial progression and relevant stimulus durations delayed mask, were counterbalanced within a block and their orders of presentation randomized.

\section{Data analysis}

Mean responses were calculated as the proportion of responses in which a participant judged the target associated with the delayed mask (either overlapping the target in the Experimental condition or on the same side as the target in the Control condition) to have lasted longer (responses were re-coded for participants making 'lasted shorter' responses). Mean responses were calculated for each participant for each of 18 conditions (nine Temporal Offset $\times$ two Mask Overlap conditions). The temporal offsets were defined as the duration of the target with a delayed mask minus the duration of the target with the simultaneous mask. For each Mask Overlap condition (Experimental and Control), participant's mean responses were fit with a cumulative normal density function (cdf) using Matlab's fminsearch to find the set of parameters (the mean, the slope, and the upper and lower asymptotes) that maximize the probability of the observed responses. Based on the derived parameters for each participant's Mask Overlap conditions, the PSE (the temporal offset at which the mean proportion of responses was equal to 50\%) was calculated.

When the masks are not overlapping the targets (Control condition), the simultaneous and delayed masks should have no impact on perception of the targets and the perceived duration of the targets should be equivalent, with the PSE equal to a temporal offset of 0. More importantly, the Experimental condition will allow us to determine whether a delayed mask can produce temporal trimming - a reduction in the perceived temporal duration of a target. Specifically, when the masks overlap their targets (Experimental condition) we predict that the perceived duration of the target with the delayed mask will be reduced. The magnitude of this reduction in perceived duration should then be evident as a positive shift in the PSE of equal magnitude.

\section{Results and discussion}

Two one-sample t-tests were conducted to determine whether the PSE values for the two Mask Overlap conditions differed from 0 . The Experimental condition's t-test was significantly different from 0 with a shift in the positive direction, $(M=$ $11.02, S E M=3.52), t(19)=3.13, p=.006, d=0.70$. The Control condition's PSE was not significantly different from $0,(M=1.72, S E M=1.67), t(19)=1.04, p=.31, d=0.23$.

Consistent with our temporal trimming prediction, in the Experimental condition, when masks overlapped their targets, the target of which the mask had a delayed offset was perceived as having been present on the screen for less time relative to the target of which the mask had a simultaneous offset. This was indicated by a positive shift $(11.0 \mathrm{~ms})$ in the 

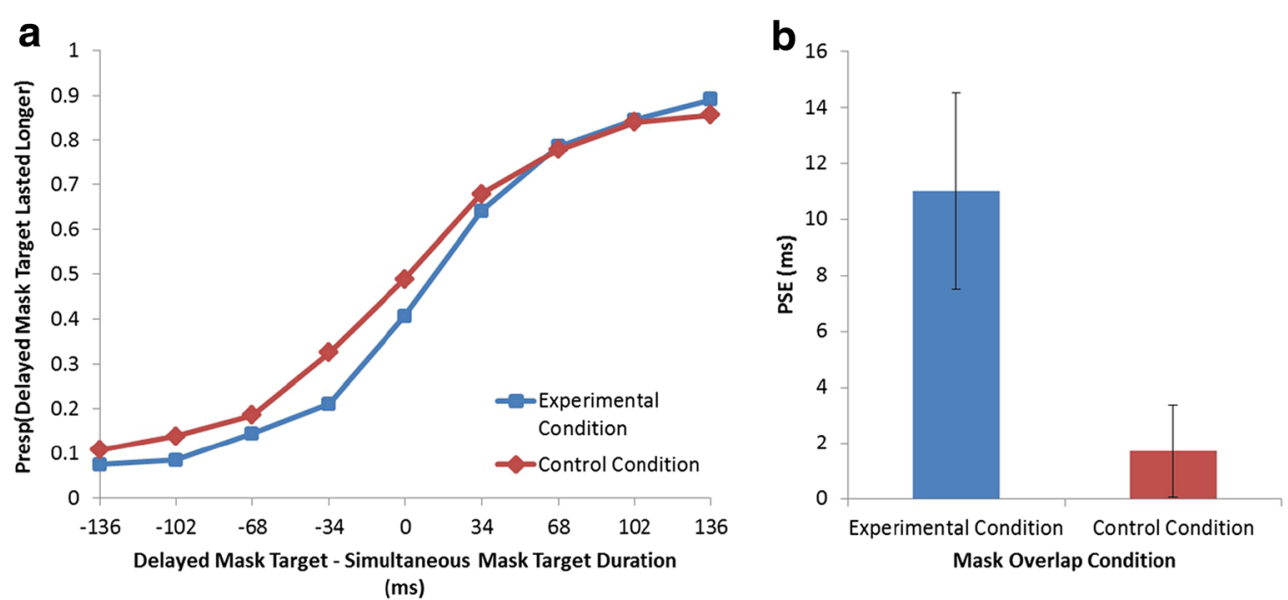

Fig. 2 Results for Experiment 1. (A) Mean proportion of responses in which participants judged the target with a delayed mask offset to have lasted longer for the nine Temporal Offsets (defined as the duration of the target with the delayed mask offset subtracted from the duration of the

PSE for the Experimental condition. These analyses lead to the conclusion that for the two targets to be perceived of equal durations, the target with a delayed mask offset needs to be presented for approximately $11 \mathrm{~ms}$ longer than the target with a simultaneous mask offset.

\section{Experiment 2}

Experiment 1 demonstrated that a four-dot mask whose offset is delayed from a target can still impact the perception of that target object even when the target is presented on the screen for durations that far exceed those of typical masking paradigms and clearly produce conscious awareness. More specifically, consistent with the hypothesis that the mechanism responsible for COM acts to shorten the window of perceptual sampling for a target object, the duration of a target with a delayed mask offset was perceived to be roughly $11 \mathrm{~ms}$ shorter than a target with a simultaneous mask offset. Further, because the temporal trimming effect was evident in the Experimental condition (when the masks overlapped the targets) but not the Control condition (when the masks overlapped distractors), it is clear that the temporal trimming effect is the function of four-dot masking and not a response bias artifact produced by the presence of asynchronous offsets in the display. Nonetheless, it is possible that when a mask with a delayed offset is present in the same spatial location as the target, as in the Experimental condition, participants' responses could have been biased by the mask's persistence. ${ }^{2}$ To address this concern and that of other research showing that twoalternative force-choice response methods can produce response biases in TOJ tasks (see Jaśkowski, 1993; Shore et al., 2001), Experiment 2 utilized a continuous response

\footnotetext{
${ }^{2}$ We thank an anonymous reviewer for providing this alternative explanation.
}

target with the simultaneous mask offset). Calculations were done separately for each of the two Mask Overlap conditions. (B) Mean point of subjective equality (PSE) values for the two Mask Overlap conditions

procedure with the goal to provide converging evidence for the conclusion of Experiment 1 that a delayed mask offset can shorten the window of perceptual sampling for a consciouslyperceived target. Specifically, we sought to replicate the temporal trimming effect using targets undergoing dynamic feature changes. Experiment 2 then used methodology similar to that of Harrison et al. (2016), such that we had participants report the orientation of a target object after approximately $1 \mathrm{~s}$ of that object changing orientation and spatial position. Instead of a forced choice method of responding, participants were asked to recreate the last perceived orientation of one of two target objects after either simultaneous or delayed maskoffset conditions. We predicted that in addition to increased guessing and increased response variability in the delayed mask-offset condition, as reported by Harrison et al. (2016), the mean reported target angle of non-guess responses should be shifted to reflect a loss of approximately $3.7-18.4 \mathrm{~ms}$ in perceived rotational motion (i.e., the lower and upper bounds of the $95 \%$ confidence interval reflecting the amount of temporal trimming observed in Experiment 1 for spatially superimposed four-dot masks).

\section{Method}

\section{Participants}

Twenty-two students from Queen's University participated in exchange for financial compensation. All had normal or corrected-to-normal vision, and were naïve to COM and the experiment's purpose.

\section{Apparatus}

The experiment was conducted on a personal computer in a dimly-lit room. Stimuli were presented with Psychophysics 


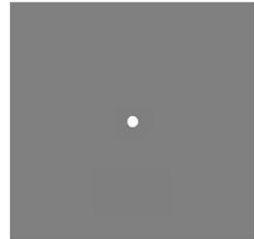

Fixation Display $500 \mathrm{~ms}$

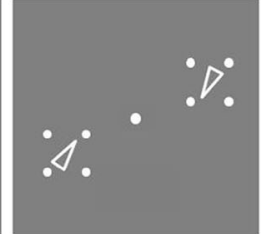

Static Display $1000 \mathrm{~ms}$

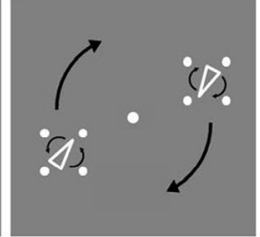

Motion Display $800-1200 \mathrm{~ms}$

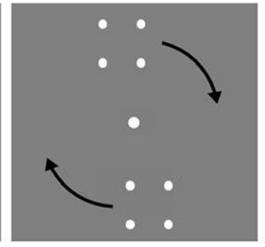

Trailing Mask Duration 0 or $300 \mathrm{~ms}$

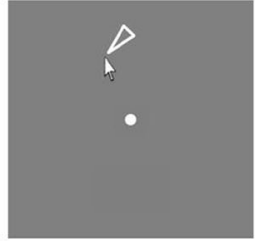

Response
Fig. 3 Example trial sequence for Experiment 2. A fixation screen was followed by an initial static display. Participants tracked both triangles as they moved in a circle around fixation with the triangles also spinning around their midpoints. Masks had either simultaneous or delayed offsets.

Toolbox version 3.0.8 (Brainard, 1997) in Matlab on a 16-in. CRT monitor. Participants responded using a keyboard.

\section{Design and procedure}

The experiment consisted of four blocks of 60 trials for a total of 240 experimental trials. The experimental trials were preceded by two blocks of ten practice trials. An example of a trial sequence is presented in Fig. 3. Each trial began with the presentation of a small white fixation point (radius $0.15^{\circ}$ ) at the center of a dark-gray background $\left(17 \mathrm{~cd} / \mathrm{m}^{2}\right)$ accompanied by two white outlines of isosceles triangles $\left(0.5^{\circ}\right)$ surrounded by four small white dots (each $0.1^{\circ}$ ) forming the corners of a square $\left(0.6^{\circ}\right.$ by $\left.0.6^{\circ}\right)$. All stimuli had $62 \mathrm{~cd} / \mathrm{m}^{2}$ luminance. The two triangles were separated at equidistant locations from fixation at two angles along an imaginary circle (radius $3.5^{\circ}$ ). The starting position of one triangle was randomly selected from $10-169^{\circ}$ and the second triangle's location was always presented at the opposite location $\left(+180^{\circ}\right)$. This initial static display was presented for $1 \mathrm{~s}$. One motion trajectory was followed by both the triangles and their surrounding masks. The target triangles underwent two forms of motion. First, the triangles' orientations rotated at $720^{\circ} / \mathrm{s}$. The direction of each triangle's rotation was selected randomly. Second, the triangles along with their masks translated along the imaginary circle that surrounded fixation at $90^{\circ} / \mathrm{s}$. The two masks' offsets were simultaneous with their targets on half the trials and delayed by $300 \mathrm{~ms}$ on the other half of trials. During delayed mask trials, the masks continued to translate at the same speed after the triangles had disappeared. The duration of both aspects of motion was randomly chosen from a set of five values between 800 and $1,200 \mathrm{~ms}$ separated by 100 -ms intervals. One of the triangles was randomly chosen to be probed for a continuous orientation response. The response interval began by presenting a mouse cursor over the final location of one of the triangles. Participants responded by moving the mouse, which drew a triangle based on the orientation of the mouse from the center of the triangle's location, and submitted their response by clicking the left mouse button. Trials were separated by a $500-\mathrm{ms}$ inter-trial interval. There were an equal
Responding was cued by the presentation of the mouse cursor over the final position of the response-relevant triangle. Participants used a mouse to match the orientation of a redrawn triangle to the final orientation of the response-relevant triangle

number of simultaneous and delayed mask-offset trials, counterbalanced within a block and presented in a random order (Fig. 4).

\section{Data analysis}

For each trial, orientation error was calculated as the difference between the probed target's actual and reported orientation in degrees. Direction of motion was accounted for by multiplying the response angle by -1 for counter-clockwise motion. Using this orientation measure, each participant's performance in the simultaneous and delayed mask-offset conditions was modelled as a weighted mixture of two distributions: (1) Guess responses, defined as randomly selecting an orientation value, and (2) Non-guess responses, defined as correctly reporting the orientation of the probed target. Guess responses were modeled using a uniform distribution and Non-guess responses were modeled using a circular Gaussian distribution - the von Mises distribution. Parameter estimates were obtained using a maximum likelihood procedure, iteratively adjusting parameters to minimize the negative LogLikelihood using the fminsearch function in Matlab. The von Mises distribution has two parameters, its mean and its concentration ( $\mathrm{K}$, a measure of spread). We converted concentration to standard deviation using a mathematical transformation described by Bays, Catalao, and Husain (2009). Thus, three parameter estimates were derived for each condition of each participant: (1) Guess responses $\left(\mathrm{P}_{\text {Guess }}\right)$ - defined as the proportion of responses accounted for by the uniform distribution, (2) Non-guess response variability (SD) - the standard deviation of the von Mises distribution, and (3) the mean shift $(\mu)$ - the displacement, in degrees, of the mean of the von Mises distribution from 0. Critically, from these three parameters it will be possible to observe both standard masking effects, evidenced by increased guessing and increased response variability, as well as a reduction in the sampling of target information, evidenced by a shift in the mean of the von Mises distribution. Specifically, if COM reduces the amount of information sampled about a target by approximately 3.7- 

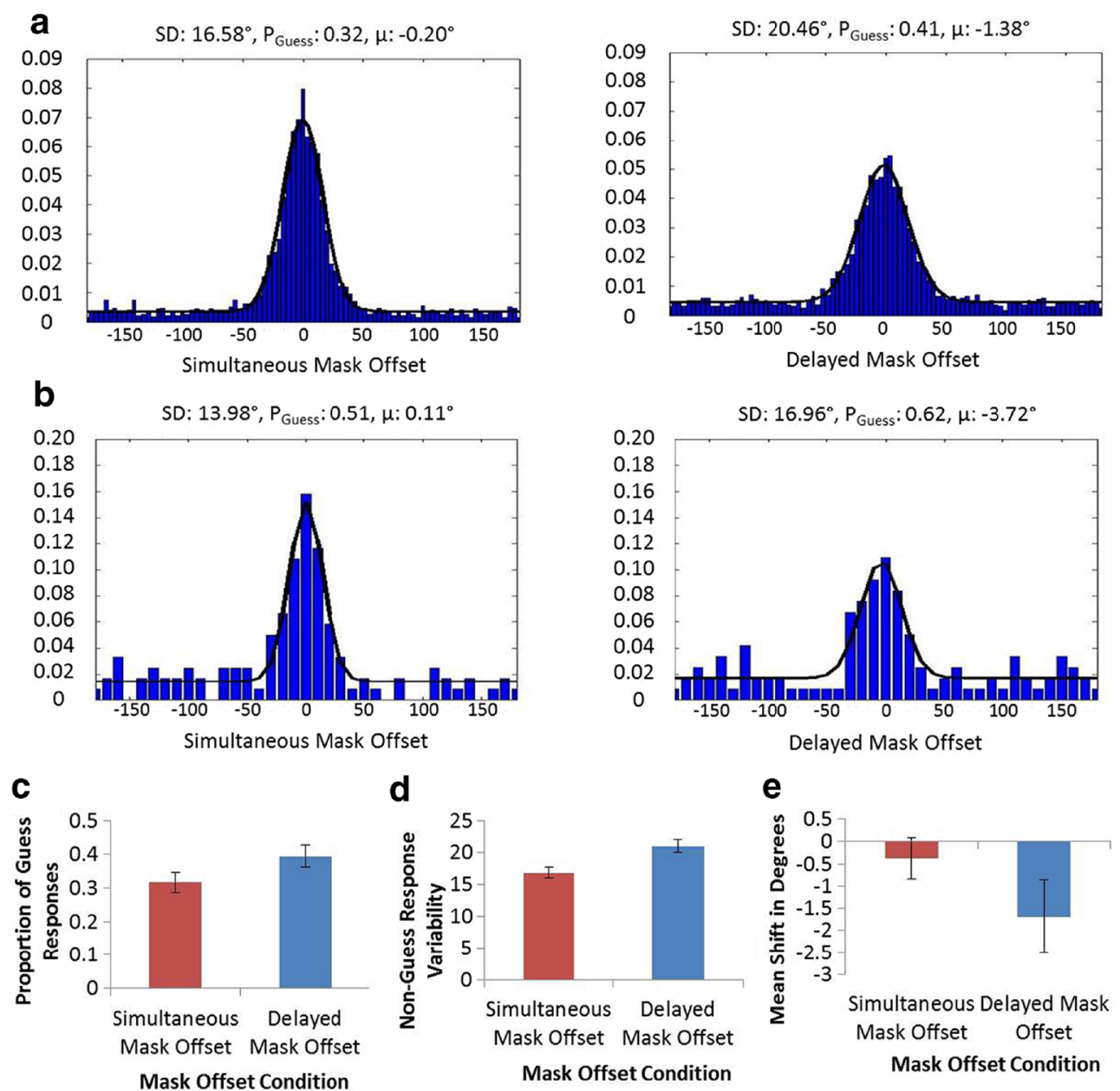

Fig. 4 Probability distributions with model fits, and results for Experiment 2. (A) Model fit for all participant data combined. (B) Model fit for a representative participant. Mean log likelihood of model fits $=160.15$. $(\mathbf{C})$ The mean proportion of guess responses $\left(\mathrm{P}_{\text {Guess }}\right)$. (D)

$18.4 \mathrm{~ms}$ then a negative shift of approximately $2.7-13.2^{\circ}$ should be observed.

\section{Results and discussion}

Two paired samples t-tests were conducted to determine whether the three parameter estimates differed between the two masking conditions (simultaneous and delayed mask offsets). $\mathrm{P}_{\text {Guess }}$ was significantly higher in the delayed mask $(M=$ $39.5 \%, S E M=3.3 \%)$ than in the simultaneous mask $(M=31.8 \%$, $S E M=3.0 \%$ ) condition, $t(21)=3.42, p=.003, d=0.73$. SD was also significantly higher in the delayed mask $(M=$ $\left.21.01^{\circ}, S E M=1.0^{\circ}\right)$ than in the simultaneous mask- $(M=$ $16.9^{\circ}, S E M=0.8^{\circ}$ ) offset condition, $t(21)=3.49, p=.002$, $d=0.75$. Two one-sample t-tests were conducted to determine whether the mean shifts for the two mask offset conditions differed from 0 . For the delayed mask offset condition, $\mu$ ( $M$ $\left.=-1.7^{\circ}, S E M=0.8^{\circ}\right)$ was significantly different from $0, t(21)$ $=2.05, p=.05, d=0.44$. In contrast, for the simultaneous
The mean non-guess response variability (SD). (E) The mean mean shift $(\mu)$ in degrees for the two mask offset conditions. Error bars represent standard errors of the mean

mask offset condition $\mu$ did not significantly differ from 0 , $t(21)=0.80, p=.43, d=0.17$.

In line with our prediction, delaying the offset of a four-dot mask surrounding a moving and rotating target object produced increases in both $\mathrm{P}_{\text {Guess }}$ and SD about a target's orientation relative to a simultaneous mask-offset condition. Importantly, these masking effects are highly similar to those observed by Harrison et al. (2016), providing additional support that the current paradigm is activating the same mechanism as seen in typical COM paradigms. More critically, in support of our hypothesis regarding reduction in target sampling by a delayed mask offset, this condition produced a negative shift in the mean of the non-guess response distribution.

Although the mean shift of the delayed mask offset condition was significantly different from 0 , the observed shift of $1.7^{\circ}$ was smaller than the lower bound of our range of predicted values $\left(2.7^{\circ}\right)$ calculated based on the temporal trimming results of Experiment 1. The smaller shift (or smaller 
reduction in the perceived duration of motion) compared to Experiment 1 could be attributed to the contribution of response bias to the temporal trimming effect in Experiment 1 (Schneider \& Bavelier, 2003). An alternative explanation is that in Experiment 2, some participants may have adopted a strategy of only paying attention to one of the two target objects during some trials. Although several lines of research have shown that COM can still produce reductions in target awareness under conditions of focused attention (Filmer, Mattingley, \& Dux, 2015; Filmer, Wells-Peris, \& Dux, 2017), this is only the case when baseline performance is not at ceiling. It is possible that the extended presentation duration of our stimuli made the Experiment 2 task relatively easy for participants who opted to pay attention to only one target for the duration of a trial, thus reducing the temporal trimming effect. This is further supported by the high average guess rates for this task, roughly $30 \%$ in the simultaneous mask offset condition.

\section{General discussion}

COM has been an important tool for exploring how the visual system resolves situations of conflict during the initial stages of object perception. However, recent work by Harrison et al., (2016), showing that COM can impair the quality of object representations, suggested that our current understanding of how COM impacts visual experience is incomplete. The present study sought to provide converging evidence for the hypothesis that COM, and more generally events that signal discontinuity about an object, prematurely terminate the sampling of perceptual information pertaining to a target object. We accomplished this by demonstrating across two experiments that delaying the offset of a four-dot mask associated with a target object acts to reduce the perceived duration of that target object. This temporal trimming effect is not only consistent with the framework proposed by Harrison et al., but also we believe extends the domain of COM research to include the modification of object representations well after their initial formation. Although there are differences between the current experimental design and standard COM paradigms, there are several reasons to believe the same mechanism is involved. First, the present results are consistent with the results of two other COM studies that examined COM following extended target presentation durations (Gellatly, Pilling, Carter, \& Guest, 2010; Guest, Gellatly, \& Pilling, 2012). For example, in Gellatly et al.'s (2010) Experiment 1, eight objects were presented for 17, 200, or $500 \mathrm{~ms}$. The target object was identified either simultaneous with its onset or $17 \mathrm{~ms}$ before its offset. Critically, when the target was not identified until $17 \mathrm{~ms}$ before its offset, awareness of the target's response-relevant feature (the location of a gap in a Landolt-C) was reduced under delayed mask-offset conditions. This reduction in target feature awareness was not observed if the target was identified at its onset in the 200- or 500-ms presentation duration conditions. Similar results were obtained by Guest and colleagues (2012) who found COM for long duration targets $(200-830 \mathrm{~ms})$ as long as the target object was not identified out of the surrounding objects until shortly before its offset ( 50 or $60 \mathrm{~ms}$ ). Second, the delayed mask condition in our Experiment 2 produced both increased $\mathrm{P}_{\text {Guess }}$ and SD of orientation responses. This suggests that in addition to a premature termination in the updating of the target's specific orientation, the delayed mask also made the information that reached awareness noisier and increased the proportion of trials in which target orientation information was completely lost. These findings mirror results of Harrison et al. (2016) using a standard COM procedure. Taken together, this suggests that the mechanism responsible for standard COM can occur for objects presented for extended durations as long as the information required for behavioral response is continuously changing across time.

There exists a debate in the study of visual masking concerning whether COM, relative to other forms of masking, is produced by a unique mechanism in the visual system ( $\mathrm{Di}$ Lollo, 2014; Põder, 2013, 2014). Although it was not the focus of this paper to explore commonalities and differences between COM and other forms of masking, it should be noted that similar patterns of increased response variability (Agaoglu, Agaoglu, Breitmeyer, \& Ogmen, 2015) and reductions in perceived duration (Cantor \& Thomas, 1976) to those found in the present study have been demonstrated by other forms of visual masking. The similarity in these findings suggests potentially overlapping mechanisms, but further research using methodologies that can assess perceived temporal duration should seek to draw more direct comparisons between different forms of masking and the effects they produce. Finally, these findings can contribute to the debate concerning whether the transition from unconscious to conscious processing is discrete or gradual in nature (Overgaard, Rote, Mouridsen, \& Ramszoy, 2006; Sergent \& Dehaene, 2004). Although a full summary of this debate is outside the scope of this paper, we believe COM provides a unique methodological opportunity for the study of conscious processing. As we have shown, COM can now be used to study impairments in how conscious representations are formed (see Salahub \& Emrich, 2016), as well as the processes involved in the updating of their representations over time. Future studies should apply COM methodologies to shed further light on the debate surrounding conscious processing.

\section{Conclusion}

Across two experiments, we assessed whether delaying the offset of a four-dot mask surrounding a target object could 
affect the perception of target information (either duration or orientation) that was changing over time. We found evidence that the delayed mask offset can reduce the perceived duration of a target's representation and cause a negative temporal shift in the perception of dynamic orientation information. We interpret this finding as the result of a mechanism disrupting a process of continuous information sampling about the target object and argue that this is the same mechanism responsible for standard COM effects.

The present results showing temporal trimming of consciously-perceived objects dovetail with evidence of reduced precision of near-threshold objects as typically presented in COM tasks (Harrison et al., 2016). To date COM has been understood as a mechanism that interrupts processing during the initial stages of object perception and leads to allor-none failures in the conscious awareness of a target object. Our results support Harrison et al.'s (2016) account of COM, such that typical COM paradigms are merely a methodology that taps into a more general mechanism responsible for terminating information sampling about an object in the event that discontinuous information is received about that object.

\section{References}

Agaoglu, S., Agaoglu, M. N., Breitmeyer, B., \& Ogmen, H. (2015). A statistical perspective to visual masking. Vision Research, 115, 2339.

Allik, J., \& Kreegipuu, K. (1998). Multiple visual latency. Psychological Science, 9(2), 135-138.

Bays, P., Catalao, R., \& Husain, M. (2009). The precision of visual working memory is set by allocation of a shared resource. Journal of Vision, 9, 1-11.

Bays, P., \& Husain, M. (2008). Dynamic shifts of limited working memory resources in human vision. Science, 321(5890), 851-4.

Brainard, D. H. (1997). The psychophysics toolbox. Spatial Vision, 10(4), 433-436.

Breitmeyer, B. G., \& Öğmen, H. (2006). Visual masking: Time slices through conscious and unconscious vision. Spatial Vision. Oxford University Press.

Cantor, N. E., \& Thomas, E. A. C. (1976). Visual masking effects on duration, size, and form discrimination. Perception \& Psychophysics, 19, 321-327.

Di Lollo, V. (2014). Reentrant processing mediates object substitution masking: Comment on Põder (2013). Frontiers in Psychology, 5, 819.

Di Lollo, V., Enns, J. T., \& Rensink, R. a. (2000). Competition for consciousness among visual events: The psychophysics of reentrant visual processes. Journal of Experimental Psychology: General, 129(4), 481-507.
Enns, J. T. (2004). Object substitution and its relation to other forms of visual masking. Vision Research, 44(12), 1321-31.

Filmer, H. L., Mattingley, J. B., \& Dux, P. E. (2015). Object substitution masking for an attended and foveated target. Journal of Experimental Psychology: Human Perception and Performance, $41(1), 6-10$.

Filmer, H. L., Wells-Peris, R., \& Dux, P. E. (2017). The role of executive attention in object substitution masking. Attention, Perception, $\{\&\}$ Psychophysics, 1-8.

Gellatly, A., Pilling, M., Carter, W., \& Guest, D. (2010). How does target duration affect object substitution masking? Journal of Experimental Psychology: Human Perception and Performance, 36(5), 1267-1279.

Goodhew, S. C., Pratt, J., Dux, P. E., \& Ferber, S. (2013). Substituting objects from consciousness: A review of object substitution masking. Psychonomic Bulletin \& Review, 20(5), 859-77.

Guest, D., Gellatly, A., \& Pilling, M. (2012). Reduced OSM for long duration targets: Individuation or items loaded into VSTM? Journal of Experimental Psychology: Human Perception and Performance, 38(6), 1541-1553.

Harrison, G. W., Rajsic, J., \& Wilson, D. E. (2016). Object-substitution masking degrades the quality of conscious object representations. Psychonomic Bulletin \& Review, 23(1), 180-186.

Jaśkowski, P. (1993). Selective attention and temporal-order judgment. Perception, 22(6), 681-689.

Lleras, A., \& Moore, C. M. (2003). When the target becomes the mask: Using apparent motion to isolate the object-level component of object substitution masking. Journal of Experimental Psychology: Human Perception and Performance, 29(1), 106-120.

Moore, C. M., \& Lleras, A. (2005). On the role of object representations in substitution masking. Journal of Experimental Psychology: Human Perception and Performance, 31(6), 1171-80.

Overgaard, M., Rote, J., Mouridsen, K., \& Ramszoy, T. Z. (2006). Is conscious perception gradual or dichotomous? A comparison of report methodologies during a visual task. Consciousness and Cognition, 15(4), 700-708.

Põder, E. (2013). Attentional gating models of object substitution masking. Journal of Experimental Psychology: General, 142(4), $1130-1141$.

Põder, E. (2014). The changing picture of object substitution masking: Reply to Di Lollo (2014). Frontiers in Psychology, 5.

Salahub, C. M., \& Emrich, S. M. (2016). Tuning perception: Visual working memory biases the quality of visual awareness. Psychonomic Bulletin \& Review, 1-6.

Schneider, K. A., \& Bavelier, D. (2003). Components of visual prior entry. Cognitive Psychology, 47(4), 333-366.

Sergent, C., \& Dehaene, S. (2004). Is consciousness a gradual phenomenon? Evidence for an all-or-none bifurcation during the attentional blink. Psychological Science, 15(11), 720-728.

Shore, D. I., Spence, C., \& Klein, R. M. (2001). Visual prior entry. Psychological Science, 12(3), 205-212.

Spencer, T. J., \& Shuntich, R. (1970). Evidence for an interruption theory of backward masking. Journal of Experimental Psychology, 85(2), 198-203.

Zhang, W., \& Luck, S. J. (2008). Discrete fixed-resolution representations in visual working memory. Nature, 453(7192), 233-5. 